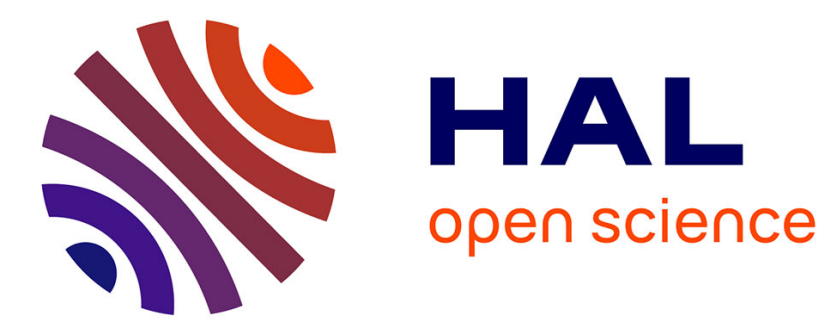

\title{
EFFET DE LA DISPERSION SUR LA PROPAGATION ACOUSTIQUE SOUS-MARINE
}

\author{
G. Loubet, J. Lacoume, Jean-Baptiste Faure, F. Vial
}

\section{To cite this version:}

G. Loubet, J. Lacoume, Jean-Baptiste Faure, F. Vial. EFFET DE LA DISPERSION SUR LA PROPAGATION ACOUSTIQUE SOUS-MARINE. Journal de Physique Colloques, 1990, 51 (C2), pp.C2945-C2-948. 10.1051/jphyscol:19902220 . jpa-00230546

\section{HAL Id: jpa-00230546 https://hal.science/jpa-00230546}

Submitted on 1 Jan 1990

HAL is a multi-disciplinary open access archive for the deposit and dissemination of scientific research documents, whether they are published or not. The documents may come from teaching and research institutions in France or abroad, or from public or private research centers.
L'archive ouverte pluridisciplinaire HAL, est destinée au dépôt et à la diffusion de documents scientifiques de niveau recherche, publiés ou non, émanant des établissements d'enseignement et de recherche français ou étrangers, des laboratoires publics ou privés. 
COLLOQUE DE PHYSIQUE

Colioque C2, supplément au $\mathrm{n}^{\circ} 2$, Tome 51, Février 1990

ler Congrès Français d'Acoustique 1990

EFFET DE LA DISPERSION SUR LA PROPAGATION ACOUSTIQUE SOUS-MARINE

G. LOUBET, J.L. LACOUME, B. FAURE et F. VIAL

Laboratoire CEPHAG, Centre d'Etudes des Phénomènes Aléatoires et Géophysiques, CNRS URA 346, BP. 46, F-38402 Saint Martin d'Hères Cedex, France

Résumé - La propagation acoustique sous-marine est caractérisée par l'existence de chemins multiples bien marqués et très stables en position. Chaque trajet est défini par un terme d'amplitude et de phase. A courte distance, ces deux paramètres sont très fluctuants ce qui peut s'expliquer par le caractère aléeatoire des réflexions de surface. A longue distance, les amplitudes varient lentement et la phase est très stable. Nous expliquons ce phénomène à partir de la dispersion et développons le calcul de ce déphasage.

Abstract - The underwater acoustic propagation is characterized by very identified, stable in range multipaths Each path is defined by a magnitude and phasis coefficient. At short range, this two parameters are fluctuant a lot, due to random surface reflexions. At long range, the magnitudes are slowly varying and the phasis is very stable. We explain this phenomenon by dispersion effect and calculate theoretically the phasis term.

\section{1 - INTRODUCTION}

Depuis une vingtaine d'années, le CEPHAG a participé à de nombreuses expérimentations, tant en Méditerranée qu'en Atlantique, et à leur dépouillement. Aussi avons nous maintenant une bonne connaissance de ce milieu. Une manipulation internationale récente regroupant un bateau émetteur et plusieurs récepteurs a eu lieu en mer du Nord (été 88). Ces nouvelles données confirment et précisent les hypothèses générales faites sur la propagation acoustique sous-marine.

Celle-ci est caractérisée par l'existence de chemins multiples très marqués et stables. Sur chaque trajet, l'élargissement fréquentiel est négligeable (seule la modulation de la houle est décelable). Plus étonnante est l'absence d'élargissement temporel notable: la propagation entre deux points se manifeste réellement par des ravons. La réponse impulsionnelle du milieu marin peut s'écrire de manière très réaliste sous la forme (réf. [2]) :

$$
h(t)=\sum_{i=1}^{N} k_{i} \delta\left(t-\tau_{i}\right) \text { avec } k_{i}=a_{i} e^{j \phi_{i}}
$$

$N$ est le nombre de trajets, $\tau_{i}$ le retard correspondant et $k_{i}$ une amplitude complexe comportant un terme d'atténuation $a_{i}$ et un terme de déphasage $\phi_{i}$. Si la position des trajets est toujours très stable, la nature du paramètre $k_{i}$ dépend beaucoup de la distance de propagation. Les échelles de temps qui nous intéressent dans cette étude vont de la minute à 1 voire 2 heures.

\section{2 - PROPAGATION COURTE DISTANCE}

Nous considèrerons une propagation à courte distance si elle est caractérisée par un trajet direct et de nombreux trajets réfléchis par la surface de la mer et le fond (réf. [1]). Ceci concerne des communications sur des distances allant de quelques $\mathrm{km}$ a $30,40 \mathrm{~km}$ (en deça de la zone de convergences des rayons qui se situe au dela de $60 \mathrm{~km}$ ). L'atténuation n'est pas le paramètre intéressant. Par contre, les amplitudes des trajets subissent des fluctuations importantes et rapides.

Ce phénomène est mis en évidence fig. ci-contre. Le signal émis consistait en une succession de séquences binaires de longueur maximale (255 digits) modulant une porteuse de $550 \mathrm{~Hz}$. Il a été reçu sur une bouée distante de $4 \mathrm{~km}$ du bateau émetteur. La figure représente la sortie du filtrage adapté des séquences, suivie d'une détection d'enveloppe donc l'évolution des amplitudes $\mathrm{a}_{\mathrm{j}}$. Le milieu est ainsi estimé toutes les $1,84 \mathrm{~s}$. On note la stabilité de la position des trajets et la très grande fluctuation des amplitudes. Les phases $\phi_{i}$ obtenues avant traitement quadratique varient elles aussi beaucoup et peuvent être considérées comme aléatoires.

Ce phénomène est dû pour une large part aux réflexions sur les facettes des vagues qui changent de taille et d'inclinaison continuellement. Le

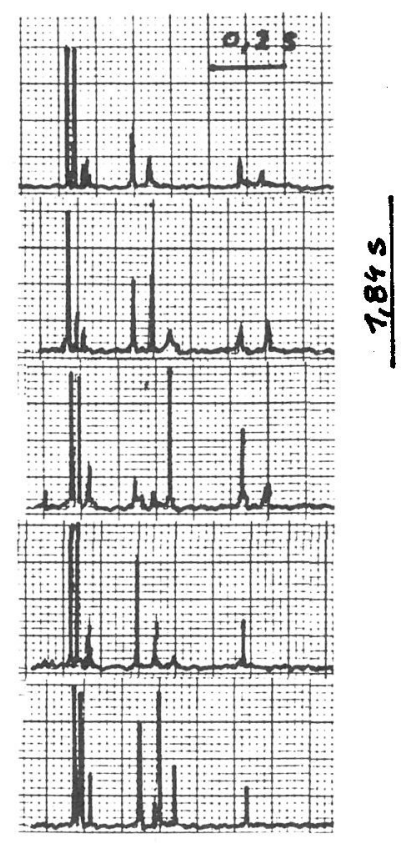


paramètre de Rayleigh, $R=2 \Pi \frac{\lambda}{\mathrm{H}} \sin \theta$ ( $\lambda$ longueur d'ordre, $H$ hauteur des vagues, $\theta$ angle d'incidence) est toujours grand (de l'ordre de l'unité) ce qui n'explique que partiellement la très grande fluctuation des amplitudes. Seules, des mers très plates, se comportant comme un miroir, n'introduiront pas de grandes fluctuations. Il convient peut-être d'ajouter à ce phénomène l'effet des bulles d'air dont l'épaisseur de la couche dépend du vent. Les effets d'interférence des reflexions de surface font que le milieu marin, dans le cas de la propagation courte distance, est un milieu très "difficile", aléatoire. Un tracé de rayons renseigne sur le nombre de chemins, leurs positions, donc sur l'étalement du canal, mais est de peu d'intérêt au niveau de la valeur des amplitudes.

\section{3 - PROPAgation lONGUE DISTANCE}

\section{3-1 Généralités}

De nombreuses expérimentations dans des gammes de fréquences allant de quelques dizaines de Hertz à $2 \mathrm{kHz}$, sur des distances de propagation de plusieurs centaines de $\mathrm{km}$, ont été dépouillées au CEPHAG. Ce type de milieu est maintenant bien connu (réf [2]) et se caractérise par :

* un petit nombre, en général, de trajets importants remarquablement stables en position (retard $\tau_{\mathfrak{i}}$ ).

* les amplitudes $\mathrm{a}_{\mathrm{i}}$ comportent une composante certaine $\mathrm{m}$, grande devant la partie aléatoire (écart type $\sigma$ petit devant $\mathbf{m}$ ). La moyenne $m$ varie lentement en fonction du temps, d'autant plus que la fréquence porteuse est faible.

* le terme de phase $\phi_{\mathrm{i}}$ est lui aussi très stable même pour des fréquences élevées (autour de $1 \mathrm{kHz}$; réf [1] , [2]), résultat en contradiction avec les modèles généralement utilisés en communication.

Il ne nous a pas été possible de mesurer un élargissement temporel notable autour d'un trajet, même pour des distances de propagation élevées. Dans les cas contraires, une étude plus fine montrait qu'on était en présence de deux trajets trés voisins. La modélisation de la réponse impulsionnelle du milieu sous forme de somme de Dirac n'est pas une approximation grossière.

En fait, plus la distance de propagation augmente, plus les signaux ont un angle d'incidence $\theta$ avec la surface de la mer petit $(\mathrm{R} \ll 1)$, plus les fluctuations dues aux reflexions sont petites (des angles $\theta$ grands correspondent à des rayons subissant beaucoup de reflexions sol-surface donc très atténués). La surface de la mer se comporte comme un miroir n'introduisant qu'un déphasage de $\pi$ sur le rayon incident. A longue distance, les chemins multiples sont dus aux réfractions des rayons acoustiques (liées aux variations de la célérité du son avec la profondeur) qui tendent à ramener l'énergie vers l'axe du chenal sonore (profondeur de célérité minimale). Nous considérons qu'une communication est à longue distance si ce phénomène de réfraction des rayons est prépondérant, soit à partir des zones de convergence $(>60 \mathrm{~km})$. La faible partie aléatoire provient d'effets résiduels de réflexion.

La partie déterministe obéit, elle, aux lois de l'absorption. Or, dans le domaine de fréquences qui intéresse la propagation acoustique sous-marine (de quelques dizaines de Hertz à $100 \mathrm{kHz}$ ), l'absorption est causée par la relaxation de composants mineurs, en particulier l'acide borique (réf [4]). A une absorption variable avec la fréquence doit être associée une variation de la vitesse des ondes avec la fréquence, le phénomène de dispersion (relations de Kramer-Kroenig).

\section{3-2 Effet de la dispersion sur la propagation (réf. [3])}

Soit une onde plane monochromatique se propageant dans le milieu supposé isotrope et homogène.

(2) $\quad a(1, t)=a_{0} \exp 2 \pi j(k l-v t) \quad$ (l distance parcourue par le rayons, $v$ fréquence)

Le nombre d'onde $k$ est fonction de la fréquence : $k(v)=k_{R}(v)+j k_{I}(v)$

$\mathrm{k}_{\mathrm{R}}(v)$ partie réelle du nombre d'onde donne l'évolution de la phase de l'onde.

$k_{I}(v)$ partie imaginaire fixe l'absorption.

La relaxation de constituants mineurs de l'eau de mer (acide borique entre $100 \mathrm{~Hz}$ et $5 \mathrm{kHz}$, sulfate le magnésium entre 5 et $100 \mathrm{kHz}$ ) se traduit par des variations de la pression en fonction de la masse volumique (réf. [5]) :

$$
\frac{\partial P}{\partial \rho}(v)=\frac{1}{1+j \frac{v}{v_{I}}}\left[c_{0}^{2}-j \frac{v}{v_{r}} c_{\infty}^{2}\right]
$$

$v_{r}$ est la fréquence de relaxation du constituant (correspondant à la constante de temps avec laquelle celui-ci revient à l'équilibre). (3) fait intervenir 2 constantes : $c_{0}$ vitesse liée aux variations de pression en fonction de la masse 
volumique $\left(c_{0}{ }^{2}=\partial \mathrm{P} / \partial \rho\right)$ dans le cas où l'onde a une fréquence très lente (le constituant évolue pour se trouver, à chaque instant, à l'équilibre) et $c_{\infty}$ dans le cas où l'onde à une fréquence très élevêe (le constituant n'a pas le temps d'évoluer).

Pour $v$ tendant vers 0 ou l'infini, le phénomène de relaxation disparaît : $\frac{\partial P}{\partial \rho}=c^{2}$ (cte).

$c_{0}$ et $c_{\infty}$ sont deux valeurs très proches. En introduisant un coefficient $E$ (très petit), le nombre d'ordre s'écrit :

$$
\text { (4) } \mathrm{k}^{2}=\frac{v^{2}}{\mathrm{c}_{0}^{2}} \frac{1}{1-\mathrm{j} \in \frac{\mathrm{v}}{v_{\mathrm{r}}-\mathrm{jv}}} \quad \text { avec } \in=\frac{\mathrm{c}_{\infty}^{2}-\mathrm{c}_{0}^{2}}{\mathrm{c}_{0}^{2}} \ll 1
$$

D'où les valeurs approchées :

$$
k_{R} \cong \frac{v}{c_{0}}\left(1-\frac{\epsilon v^{2}}{2\left(v_{r}^{2}+v^{2}\right)}\right) \quad k_{I} \cong \frac{\epsilon}{2 c_{0}} \frac{v^{2} v_{r}}{v_{r}^{2}+v^{2}}
$$

Soit un signal à bande étroite autour d'une porteuse $v_{0}$ et une distance de propagation 1 . Développons la partie réelle du nombre d'onde au ler ordre:

(6) $\quad k_{R}(v) \equiv k_{R}\left(v_{\emptyset}\right)+\left(v-v_{0}\right) \frac{d k_{R}}{d v}\left(v_{0}\right)$

En introduisant les notions classiques de vitesse de phase et vitesse de groupe, on pose :

$$
\begin{aligned}
& \tau_{\rho}\left(v_{0}\right)=1 \frac{k_{R}\left(v_{0}\right)}{v_{0}} \text { retard lié à la vitesse de phase } c_{p}=\frac{v}{k_{R}} \\
& \tau_{g}\left(v_{0}\right)=1 \frac{d k_{R},}{d v}\left(v_{0}\right) \text { retard lié à la vitesse de groupe } c_{g}=\frac{d v}{d k_{R}} \\
& k_{R} \cong v \tau_{g}+v_{0}\left(\tau_{p}-\tau_{g}\right)
\end{aligned}
$$

Lorsque le nombre d'ordre $k$ est complexe, il est nécessaire que $k_{I}(v) \ll k_{R}(v)$ pour que le concept de vitesse de groupe reste valable et s'identifie à la vitesse de propagation de l'énergie (réf. [6]). C'est le cas du milieu marin qui ne comporte pas de bande d'absorption (qui nécessiterait l'utilisation de la notion de vitesse du signal). Développer $\mathrm{k}_{\mathrm{I}}(\mathrm{v})$ au premier ordre correspondrait à un développement au second ordre de $\mathrm{k}_{\mathrm{R}}(\mathrm{v})$. Aussi est-il justifié de considérer dans la bande du signal émis :

(8) $\mathbf{k I}_{\mathrm{I}}(\mathrm{v})=\mathrm{k}_{\mathrm{I}}\left(\mathrm{v}_{0}\right)=$ constante .

Sur un trajet de propagation, la fonction de transfert du milieu est :

(9) $\quad H(v)=\exp 2 \pi j k(v) l=\exp \left(2 \pi j k_{R} 1-2 \pi k_{I} 1\right)$

L'atténuation correspond au terme $\exp \left(-2 \pi \mathrm{k}_{\mathrm{I}}\left(v_{0}\right) 1\right)=\mathrm{a}\left(\mathrm{v}_{0}\right)$.

(10) $\mathrm{H}(v)=\mathrm{a}\left(v_{0}\right) \exp 2 \pi \mathrm{j} 1\left(v \tau_{\mathrm{g}}+v_{0}\left(\tau_{\mathrm{p}}-\tau_{\mathrm{g}}\right)\right)$

Pour un trajet, la réponse impulsionnelle du milieu est la Transformée de Fourier inverse de $H(v)$ :

$$
h(t)=a\left(v_{0}\right) e^{-j \phi \delta\left(t-\tau_{g}\right)} \text { avec }
$$

$$
\phi=2 \pi v_{0}\left(\tau_{\mathrm{p}}-\tau_{\mathrm{g}}\right)=\phi_{\mathrm{T}}\left[1-\frac{v_{0}}{\mathrm{k}_{\mathrm{R}}\left(v_{0}\right)} \frac{\mathrm{dk}}{\mathrm{dv}}\left(v_{0}\right)\right]
$$

$\phi_{T}=2 \pi k_{R}\left(v_{0}\right) l$ est la variation de phase totale issue de la propagation.

Pour un milieu non dispersif, $\mathrm{k}_{\mathrm{R}}=\frac{\mathrm{v}}{\mathrm{c}}$ et $\phi=0$.

Ce modèle théorique correspond bien aux résultats expérimentaux: pas d'élargissement temporel et une réponse complexę introduisant un déphasage. Pour un signal passe-bande, la modélisation ci-dessus est valable tant qu'on 
peut assimiller la courbe $\mathbf{k}_{\mathbf{R}}(v)$ à sa tangente au point $v_{0}$. Ceci n'est pas très restrictif car la courbe est voisine d'une droite $(v / c)$.

Pour un signal émis $s(t)=m(t) \exp \left(2 \pi j v_{0} t\right)$, le signal reçu sera la convolution de $s(t)$ par $h(t)$ :

(13) $\quad r(t)=a\left(v_{0}\right) m\left(t-\tau_{g}\right) \exp \left[j\left(2 \pi v_{0}\left(t-\tau_{g}\right)+\phi\right]\right.$

$\mathrm{a}\left(v_{0}\right)$ représente l'atténuation, $\phi$ est un terme de déphasage entre l'enveloppe et la porteuse, déphasage relevé dans de nombreuses expérimentations. A partir de (5) et (12), on trouve pour ce déphasage :

$$
\phi=\phi T \varepsilon \frac{x^{2}}{\left(1+x^{2}\right)^{2}} \text { avec } x=\frac{v}{v_{r}}
$$

$\phi$ est maximum pour $v=v_{r} . \phi$ tend vers 0 pour $v$ tendant vers 0 ou l'infini.

L'absorption $\mathrm{a}(\mathrm{v})$ est en $\mathrm{dB} / \mathrm{m}$ donné par (5):

$a(v)=20 \pi \log e \frac{\epsilon}{c_{0}} \frac{v^{2} v_{r}}{v_{r}^{2}+v^{2}} \underset{v \rightarrow \infty}{\longrightarrow} 20 \pi \log e \frac{\epsilon v_{r}}{c}$

d'où $\epsilon=\frac{c_{0} a(\infty)}{20 \pi \log \text { e } v_{r}} \quad$ et pour une distance 1 de propagation: $\quad \phi=\frac{1 \mathrm{a}(\infty)}{10 \log \mathrm{e}} \frac{\mathrm{x}^{3}}{\left(1+\mathrm{x}^{2}\right)^{2}}$

Pour des fréquences comprises entre $100 \mathrm{~Hz}$ et $5 \mathrm{kHz}$ correspondant à la relaxation de l'acide borique $\left(v_{\mathrm{r}}=1 \mathrm{kHz}, \mathrm{a}(\infty)=10^{-4} \mathrm{~dB} / \mathrm{m}\right)$, on obtient pour une distance $\mathrm{l}=100 \mathrm{~km}$ le tableau suivant :

\begin{tabular}{|c|c|c|c|c|c|}
\hline$v$ & $100 \mathrm{~Hz}$ & $300 \mathrm{~Hz}$ & $1 \mathrm{kHz}$ & $1,73 \mathrm{kHz}$ & $5 \mathrm{kHz}$ \\
\hline$\phi$ & $0,41^{\circ}$ & $9,4^{\circ}$ & $103^{\circ}$ & $134^{\circ}$ & $76^{\circ}$ \\
\hline
\end{tabular}

On voit que les déphasages sont loin d'être négligeables et peuvent être facilement estimés à partir des formules précédentes.

\section{4 - CONCLUSIONS}

La caractéristique essentielle du milieu marin est l'existence de chemins multiples. La réponse impulsionnelle correspondante est donnée en (1).

A courte distance, c'est le phénomène de réflexion fond-surface qui prédomine et les paramètres $a_{k}$ et $\phi_{k}$ sont fortement aléatoires. Un tracé de rayon ne peut fournir qu'une idée de la position des trajets. Pour une communication, le traitement approprié sera quadratique (corrélation, détection d'enveloppe suivi d'un filltrage "adapté" à l'enveloppe du milieu).

A longue distance, le phénomène de réfraction des rayons acoustiques prédomine. Le milieu peut être considéré comme quasi certain. Les amplitudes et les positions des trajets peuvent être prédits par tracé de rayons. La dispersion explique le terme de phase $\phi_{\mathrm{k}}$ qui peut être estimé en fonction de la distance de propagations et de la fréquence porteuse. Pour une communication, un traitement optimal devrait comporter un filtrage adapté à ce modèle déterministe complexe.

Ce travail a été réalisé avec le concours de la Direction des Constructions Navales.

\section{Références}

[1] URICK R.J. : Principles of Underwater Sound. Mc Graw. Hill, 1975.

[2] LOUBET G., PALLAS M.A. : Caractérisation du milieu marin en tant que canal acoustique. J. Acoustique, 1, $129-134,1988$.

[3] LACOUME J.L., LOUBET G. : Sur le déphasage entre l'enveloppe et la porteuse en acoustique sous-marine. 12ème Colloque GRETSI, 897-900, 1989.

[4] CLAY S., MEDWIN H. : Acoustical Oceanography. Wiley Interscience, 1977.

[5] LANDAU L., LIFCHITZ E. : Mécanique des Fluides. Ed. Mir, Moscou, 1971.

[6] QUEMADA D. : Ondes dans les plasmas. Hermann, Paris, 1968. 